\title{
Inner City
}

National Cancer Institute

\section{Source}

National Cancer Institute. Inner City. NCI Thesaurus. Code C18067.

The older and more populated and (usually) poorer central section of a city. 